\title{
Variations
}

Variations

Revue internationale de théorie critique

$12 \mid 2008$

Wärmestrom

\section{À propos de la personnalité autoritaire}

Theodor W. Adorno, Études sur la personnalité autoritaire

\section{Julien Bordier}

\section{OpenEdition}

\section{Journals}

Édition électronique

URL : https://journals.openedition.org/variations/246

DOI : 10.4000/variations.246

ISSN : 1968-3960

Éditeur

Les amis de Variations

\section{Édition imprimée}

Date de publication : 21 décembre 2008

Référence électronique

Julien Bordier, «À propos de la personnalité autoritaire », Variations [En ligne], 12 | 2008, mis en ligne le 01 janvier 2012, consulté le 21 septembre 2021. URL : http://journals.openedition.org/variations/246 ; DOI : https://doi.org/10.4000/variations.246

Ce document a été généré automatiquement le 21 septembre 2021

Les ami•e•s de Variations 


\section{À propos de la personnalité autoritaire}

Theodor W. Adorno, Études sur la personnalité autoritaire

Julien Bordier

\section{RÉFÉRENCE}

Theodor W. Adorno, Études sur la personnalité autoritaire, Paris, Allia, 2008 (1950), 435 p.

\section{NOTE DE L'ÉDITEUR}

Première publication sur www.theoriecritique.com, « Wärmestrom, le courant chaud en sciences humaines », Hiver 2008/2009, pp. 75-78

1 Les Études sur la personnalité autoritaire étaient une des principales contributions de Theodor W. Adorno à ne pas encore avoir été traduites en français. Elle s'inscrit dans la lignée des ouvrages du théoricien de l'Ecole de Francfort : proposer une pensée critique et non autoritaire. L'objet de la recherche menée est précisément le caractère autoritaire et les relents fascistes existant chez les individus. Ces textes, écrits durant l'exil américain d'Adorno, font partie du premier volume de The autoritarian personnality, large enquête éditée notamment par Max Horkeimer avec le soutien du American Jewish Committee au lendemain de la seconde guerre mondiale. La question sous-jacente est bien sûr de réussir à percevoir ce qui a rendu possible l'adhésion de masse au fascisme. Mais il ne s'agit pas d'étudier des sujets qui adhéreraient ouvertement au programme nazi par exemple. Au contraire, le problème consiste à déceler, dans une société reconnue comme démocratique, la possible adhésion des individus à des idées non-démocratiques. Les premières lignes de l'ouvrage éclairent sans ambiguïté ce point :

« Nous disons potentiellement parce que nous n'avons pas étudié d'individus qui étaient ouvertement fascistes, ou qui appartenaient à des organisations fascistes connues. À l'époque où nous avons recueilli la plupart de nos données, le fascisme 
venait de perdre la guerre et, par conséquent, nous ne pouvions pas nous attendre à rencontrer des sujets qui s'y seraient ouvertement identifiés; cependant nous n'eûmes pas de difficulté à trouver des sujets dont la vision du monde était de nature à indiquer qu'ils auraient été prêts à accepter le fascisme au cas où il serait devenu un mouvement social puissant et respectable.» (p. 7) Rares sont ceux qui expriment ouvertement leur adhésion aux idées d'extrême droite. Pourtant, la dernière campagne présidentielle s'est disputée entre les candidats sur les thèmes favoris de cette dernière: insécurité, immigration et retour à l'autorité traditionnelle. Quant au précédent scrutin présidentiel, il avait été marqué par la présence d'un candidat d'extrême-droite au second tour. Un Ministère de l'identité nationale a été créé et l'obsession sécuritaire est devenu un principe de gouvernement dans un relatif consensus. Dans ce contexte, comprendre quels schémas sociopsychologiques font qu'un individu accepte l'autorité et adhère à des idées antidémocratiques est éclairant.

3 Au sein de l'œuvre d'Adorno, les Études sur la personnalité autoritaire ont la particularité d'être une enquête sociologique. Elles permettent de découvrir la réflexion du théoricien de l'Ecole de Francfort sous un autre visage que celui d'une conceptualisation philosophique parfois difficile à aborder. Au lieu de construire une analyse tout à fait empirique, l'enquête cherche à montrer que les dispositions autoritaires, qui mènent aux dispositions fascistes - c'est-à-dire finalement aux risques d'adhésion à la propagande et aux idées fascistes - ne sont pas déterminées socialement. Elles sont davantage liées à l'expression de la personnalité et à des structures mentales. Plus que d'appartenance générale de classe, il est question de milieux très précis, de processus de socialisation et de phénomènes psychologiques inconscients. L'adhésion au fascisme n'est pas liée à une analyse de sa propre situation et à des revendications rationnelles.

4 Cette enquête peut donc être qualifiée de défi sociologique. Quelle méthode mettre en place pour comprendre ce qui influence un individu dans son adhésion au fascisme et comment le déceler chez un individu qui ne dira jamais ouvertement cette adhésion, et qui ne la ressent même pas clairement lui-même? Un questionnaire est présenté à différents groupes d'individus particuliers des États-Unis. Entre autres groupes, des ouvriers syndiqués dans différentes organisations et des ouvriers incarcérés, des femmes de la classe moyenne membres d'une association parents-enseignants et d'autres membres d'une association ecclésiastique, des étudiants en cours d'art oratoire et d'autres en cours externe de psychologie, les membres de différents clubs sont interrogés :

« Tout au long de cette étude on a cherché à obtenir différents types de sujets afin de garantir une grande variabilité d'opinion et d'attitudes et une couverture adéquate des facteurs censés influencer l'idéologie. » (p. 40)

Les données recueillies sur les plus de 2000 sujets sont d'abord empiriques. Une liste de propositions est soumise aux sujets de l'enquête, auxquelles ils doivent donner leur accord ou leur désapprobation. Mélangées dans la liste, les propositions sont liées à des thèmes psycho-sociologiques. Ces thèmes sont par exemple le conventionnalisme, la soumission à l'autorité, l'agressivité autoritaire, l'anti-intraception, la superstition et la stéréotypie, le rapport à la sexualité, etc. En fonction des réponses « d'accord » ou « pas d'accord ", les penchants autoritaires et anti-démocratiques sont notés et classés sur une échelle $F$, comme fascisme. Cette échelle $F$ est elle-même construite et enrichie par d'autres échelles: A-S comme antisémitisme, CPE comme conservatisme politico- 
économique et $\mathrm{E}$ comme ethnocentrisme. Les premières conclusions de cette analyse empirique montrent que le potentiel d'adhésion au fascisme traverse de manière quasiment homogène toutes les couches de la société. Les écarts importants ne se remarquent qu'en fonction de groupes très précis. Les typologies sociales classiques sont bouleversées et c'est dans les milieux où sont le mieux admises et valorisées l'introspection et la réflexion, où est le moins directement subi l'autoritarisme, que le fascisme semble avoir le moins de prise sur les individus.

6 L'autre partie de l'enquête s'appuie sur des «entretiens cliniques ». Des individus déjà soumis au questionnaire sont interviewés en fonction des scores qu'ils ont reçus. L'objectif est alors, en les laissant s'exprimer le plus librement possible, de comprendre quels sont les schémas psychologiques cohérents qui rendent les individus perméables ou non à l'idéologie fasciste. Les extraits de ces entretiens sont cités en étant eux-même regroupés par thèmes et figures, ce qui permet au lecteur de comprendre quels sont les points où se manifestent particulièrement la personnalité autoritaire. On voit alors que le potentiel fasciste est d'abord lié à une peur de l'inconnu, une désubjectivisation et la nonexpérience de l'individu. Parmi ces études qualitatives de l'idéologie, le chapitre sur l'antisémitisme est éloquent: plus la figure du juif relève du fantasme et de la nonexpérience chez les personnes interrogées, plus s'expriment des propos anti-juifs allant même jusqu'à évoquer l'extermination. La cohérence personnelle, c'est-à-dire le schéma individuel permettant l'adhésion à l'idéologie, bien qu'irrationnelle, est mise en évidence.

Ce sont donc des processus particuliers qui font émerger la personnalité autoritaire antidémocratique - rejetant les minorités, sanctifiant l'ordre, se réfugiant dans la superstition, projetant sur les autres ses propres pulsions - et non pas l'appartenance à une classe sociale. Les déterminismes sociaux sont alors mis de côté et l'enquête montre l'importance de critères psychologiques, éclairés par les concepts de la psychanalyse.

8 Autre conclusion intéressante qui transparaît des Etudes sur la personnalité autoritaire: alors que le fascisme crée un schéma cohérent dans la pensée des «individus à haut score ", c'est-à-dire ceux dont le potentiel d'adhésion au fascisme est le plus élevé, l'antifascisme des « individus à bas score » ne laisse pas entrevoir la même constance de points communs entre les individus. Cela laisse à penser que toute idéologie est autoritaire et n'offre comme issue que l'adhésion plutôt que l'autonomie des individus.

$9 \quad$ Les enjeux de cet ouvrage sont nombreux. Il montre que de multiples types d'écoutes sociologiques sont nécessaires et qu'il est pertinent de déplacer l'analyse de groupes sociaux généraux vers une attention particulière à des traits personnels propres aux individus. Les conclusions de l'enquête montrent que la personnalité autoritaire n'est pas directement liée à une appartenance de classe mais à des traits de la personnalité et des modes de socialisation propres à des milieux précis. Plus qu'en termes de déterminants sociaux et d'appartenance de classe, il convient donc de penser des processus en mouvement dans lesquels les concepts psychanalytiques doivent être mobilisés.

Dans les Études sur la personnalité autoritaire, l'enjeu méthodologique devient un enjeu politique. Déceler les penchants autoritaires et anti-démocratiques est un moyen d'affiner la critique de l'autoritarisme où qu'il se manifeste, d'observer qu'il est lié aux mutilations personnelles des individus, de comprendre qu'il repose sur la difficulté des individus à construire leurs expériences propres. On est également bien sûr tenté de penser qu'une telle théorisation est un moyen de se débarrasser de l'autorité subie mais aussi de ses propres penchants autoritaires. 
11 Comme nous l'écrivions, la tentation fasciste reste une question d'actualité. Elle semble même être régulièrement actualisée sans dire son nom. Les événements historiques ont montré que les colères sociales engendrées par les grandes crises économiques peuvent se politiser dans des mouvements réactionnaires de masse. Les Études sur la personnalité autoritaire donnent donc aujourd'hui particulièrement à réfléchir. Dans quelles brèches et failles les individus arrivent-ils à construire et à créer des expériences autonomes et démocratiques? Existe-t-il des courants oppositionnels qui refusent l'autorité et se proposent de créer des alternatives politiques à partir d'une critique du pouvoir en général ? Comment ces diversités d'expériences subjectives peuvent se retrouver dans des mouvements qui seraient assez vigoureux intellectuellement pour freiner les poussées totalitaires?

\section{AUTEURS}

\section{JULIEN BORDIER}

Doctorant en sociologie 